\title{
Tunable terahertz radiation from graphene induced by moving electrons
}

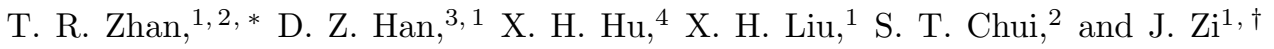 \\ ${ }^{1}$ Department of Physics, Key laboratory of Micro and Nano Photonic Structures (Ministry of Education), \\ and Key Laboratory of Surface Physics, Fudan University, Shanghai 200433, P. R. China \\ ${ }^{2}$ Bartol Research Institute, University of Delaware, Newark, Delaware 19716, USA \\ ${ }^{3}$ Department of Applied Physics, College of Physics, \\ Chongqing University, Chongqing 400044, P. R. China \\ ${ }^{4}$ Department of Materials Science and Laboratory of Advanced Materials, \\ Fudan University, Shanghai 200433, P. R. China
}

(Dated: March 8, 2022)

\begin{abstract}
Based on a structure consisting of a single graphene layer situated on a periodic dielectric grating, we show theoretically that intense terahertz $(\mathrm{THz})$ radiations can be generated by an electron bunch moving atop the graphene layer. The underlying physics lies in the fact that a moving electron bunch with rather low electron energy $(\sim 1 \mathrm{keV})$ can efficiently excite graphene plasmons $(\mathrm{GPs})$ of $\mathrm{THz}$ frequencies with a strong confinement of near-fields. GPs can be further scattered into free space by the grating for those satisfying the phase matching condition. The radiation patterns can be controlled by varying the velocity of the moving electrons. Importantly, the radiation frequencies can be tuned by varying the Fermi level of the graphene layer, offering tunable $\mathrm{THz}$ radiations that can cover a wide frequency range. Our results could pave the way toward developing tunable and miniature $\mathrm{THz}$ radiation sources based on graphene.

PACS numbers: 78.67.Wj, 73.20.Mf, 42.72.-g
\end{abstract}

Graphene photonics and optoelectronics have attracted intense research interest in recent years [1]. This is because graphene possesses exceptional electronic and optical properties due to its unique electronic band structure, i.e., the existence of Dirac cones [2. Indeed, a variety of novel applications such as broadband photodetectors, optical modulators, and ultra-fast lasers have been proposed [1]. Interestingly, graphene can support plasmons with frequencies in terahertz $(\mathrm{THz})$ and midinfrared regimes [3]. Compared with surface plasmons in noble metals [4, GPs exhibit remarkable properties such as deep subwavelength, extreme light confinement, and low Ohmic losses with a further advantage of being tunable through electrostatic gating or chemical doping [5. 6. These features make graphene a promising material for active plasmonic devices [6, which could find applications in transformation optics [7, metamaterials [8, and light harvesting [9] and concentrating [10].

$\mathrm{THz}$ radiation with frequencies from $0.1 \mathrm{THz}$ to $30 \mathrm{THz}$ has attracted increasing attention due to its wide range of potential applications [11. However, a lack of desired sources of $\mathrm{THz}$ radiation limits the realization of such applications. During the past decade, many approaches including optically pumped solid-state devices, quantum cascade lasers, diodes, and free-electron devices, have been investigated for the development of $\mathrm{THz}$ sources [12. Free-electron $\mathrm{THz}$ sources, wherein radiation occurs as moving electrons interact typically with a perturbing element [13 15, are of particular interest owing to their high power and continuous tunability by varying electron energies [12. However, the difficulty in reducing their size while retaining their broad tunability remains a great challenge for the applications of such sources.

In this Letter, we show theoretically that intense $\mathrm{THz}$ radiations can be generated by a moving electron bunch atop a graphene layer situated on a periodic dielectric grating. We demonstrate the key role of GPs excited by the moving electron bunch in the radiation process. In particular, we find that the radiation intensity is strongly enhanced due to low losses and high confinement of the excited GPs. The radiation patterns and frequencies can be tuned by varying the electron velocity or the Fermi level of the graphene layer. Our results may open up a new route to develop miniature and tunable free-electron $\mathrm{THz}$ radiation sources based on graphene.

We start by considering an electron bunch moving atop a graphene layer on a dielectric substrate. Suppose the electron bunch moves at a constant velocity $v$ in the $x$ direction atop the graphene layer by a distance $b$. For simplicity, the electron bunch is assumed to be uniform along $y$ with a line charge density $\rho$. The moving electron bunch can be viewed as a source with a current density $\mathbf{J}(\mathbf{r}, t)=\hat{\mathbf{x}} \rho v \delta(z-b) \delta(x-v t)$, which may induce a transverse-magnetic electromagnetic (EM) wave of a form $e^{i\left(k_{x} x+k_{z}|z-b|\right)}$ with its magnetic field polarized along $y$ in the frequency domain [16], representing a plane wave of a wave vector $k_{x} \hat{\mathbf{x}}+\operatorname{sgn}(z-b) k_{z} \hat{\mathbf{z}}$, where $k_{x}=\omega / v, k_{z}=\omega \sqrt{1 / v_{p}^{2}-1 / v^{2}}$, and $\omega$ and $v_{p}$ are the angular frequency and phase velocity of light in an ambient medium, respectively. Obviously, Cherenkov radiation occurs when $v>v_{p}$ [17, whereas no radiation is expected when $v<v_{p}$ since $k_{z}$ is purely imaginary and therefore the induced EM wave is evanescent.

Doped or gated graphene can support GPs that prop- 

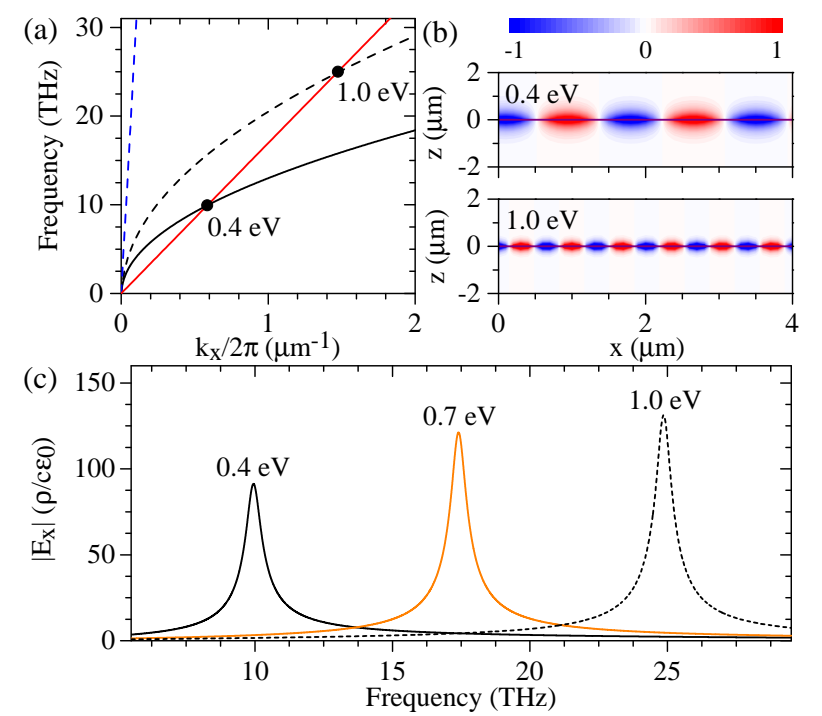

FIG. 1. (Color online) Excitation of GPs in a graphene layer at $z=0$ on a dielectric substrate with $\varepsilon_{d}=4$ by an electron bunch moving with constant velocity $v$ in the $x$ direction and at $z=b$. The graphene has an intrinsic relaxation time of $\tau=0.6$ ps throughout this work. (a) Dispersion curves of GPs at different $E_{F}$ (black lines). The red solid line shows the electron beam line $\omega / k_{x}=v$ with $v=0.057 c$, and the blue dashed line represents the light line $\omega / k_{x}=c$, where $c$ is the light speed in air. (b) Distribution of field $\operatorname{Re}\left(E_{x}\right)$ for two GP modes [dots in (a)]. (c) $\left|E_{x}(z=0)\right|$ versus frequency for $v=0.057 c$ and $b=0.1 \mu \mathrm{m}$ at different $E_{F}$.

agate along a graphene layer with associated EM fields strongly confined near its surface [3, 5], as shown in Fig. 1(b). GPs possess slow phase velocity [5, which is approximated by $v_{\mathrm{gp}} / c \simeq \frac{4 \alpha}{1+\varepsilon_{d}} \frac{E_{F}}{\hbar \omega}$ in the nonretarded regime $\left(k_{\mathrm{gp}} \gg \omega / c\right)$ [5], where $k_{\mathrm{gp}}$ is the GP wavevector, $E_{F}$ is the Fermi energy, $\varepsilon_{d}$ is the permittivity of the dielectric substrate, $\alpha$ is the fine-structure constant, and $c$ is the light speed in air. Note that $v_{\mathrm{gp}} / c$ can be of the order of $10^{-1} \sim 10^{-2}$ and further tuned by varying $E_{F}$ at $\mathrm{THz}$ frequencies, suggesting that graphene can be used as a tunable slow-wave structure.

When the electron bunch moving atop a graphene layer, the induced evanescent EM wave can excite GPs if satisfying the phase-matching condition [18], namely

$$
k_{\mathrm{gp}}(\omega)=\omega / v .
$$

Clearly, Eq. (1) is equivalent to $v_{\mathrm{gp}}(\omega)=v$, which is satisfied at intersections of GP dispersion curves with electron beam lines. Thus, the GP modes excited are given by $\omega_{\mathrm{gp}} \simeq \frac{4 \alpha}{1+\varepsilon_{d}} \frac{E_{F}}{\hbar v / c}$. As shown in Fig. 1(a), for $E_{F}=0.4 \mathrm{eV}$, the frequency of the excited GP is about $10 \mathrm{THz}$ with $v=0.057 \mathrm{c}$ (corresponding to rather low electron energy $0.832 \mathrm{keV}$ ). The excited GP frequency can be tuned by adjusting $E_{F}$, e.g., about $24.9 \mathrm{THz}$ for $E_{F}=1.0 \mathrm{eV}$.

To give a quantitative description, we solve rigorously
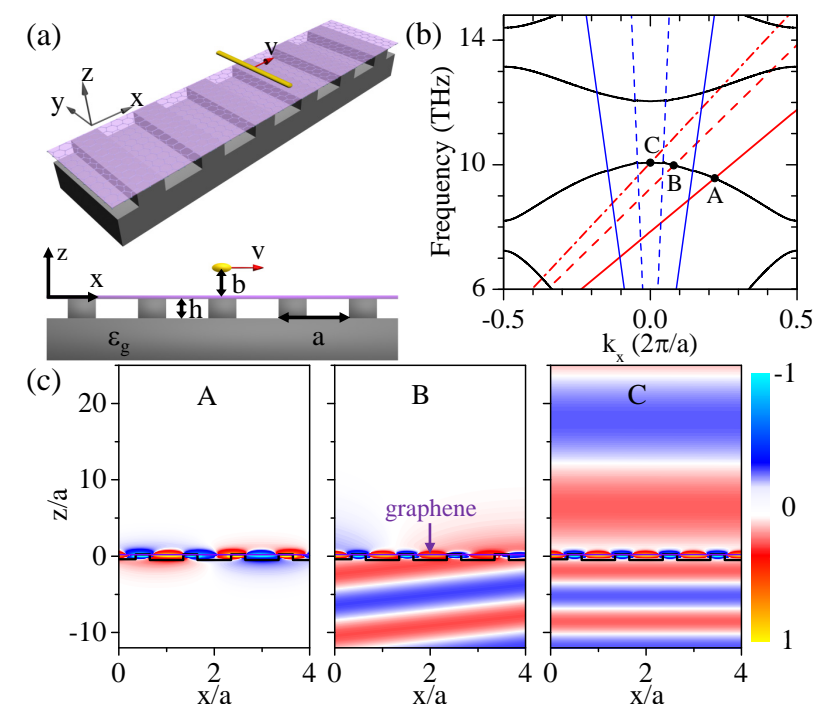

FIG. 2. (Color online) (a) 3D view (upper panel) and side view (lower panel) of an electron bunch moving atop a graphene layer on an 1D dielectric grating. (b) Dispersion curves (black solid lines) of GPs for the structure in (a). Red solid, dashed, and dash-dotted lines show folded electron beam lines $\omega /\left(k_{x}+2 \pi / a\right)=v$ with $v=0.034 c, 0.04 c$, and $0.0436 c$, respectively. Blue dashed and solid lines represent light lines $\omega / k_{x}=c$ (air) and $\omega / k_{x}=c / \sqrt{\varepsilon_{\mathrm{g}}}$ (substrate), respectively. (c) Distribution of field $\operatorname{Re}\left(E_{x}\right)$ at frequencies of modes $\mathrm{A}, \mathrm{B}$, and $\mathrm{C}$ excited by the electron bunch with corresponding $v$ in (b) and $b=0.1 \mu \mathrm{m}$. The black solid lines in (c) depict the profile of the grating.

Maxwell equations in the frequency domain in order to obtain the EM fields induced by the moving electron bunch. By considering the EM fields associated with the moving electron bunch as incident fields upon the graphene layer [19, 20, reflection and transmission should be expected, yielding the total electric fields as [16.

$$
E_{x}(\mathbf{r}, \omega) \equiv \zeta e^{i k_{x} x} \begin{cases}e^{-\gamma_{0}|z-b|}-r e^{-\gamma_{0}(z+b)}, & z>0 \\ t \eta e^{-\gamma_{0} b} e^{\gamma_{2} z}, & z<0\end{cases}
$$

where $\zeta=-i \frac{\rho}{2 c \varepsilon_{0}} \frac{\gamma_{0}}{k_{0}}, r=\frac{1-\eta+\xi}{1+\eta+\xi}$ and $t=\frac{2}{1+\eta+\xi}$ are reflection and transmission coefficients of the graphene layer respectively, $\eta=\gamma_{2} /\left(\varepsilon_{d} \gamma_{0}\right), \xi=i \sigma_{\mathrm{gp}} \gamma_{2} /\left(\varepsilon_{0} \varepsilon_{d} \omega\right)$, $\gamma_{0}=\sqrt{{k_{x}}^{2}-k_{0}{ }^{2}}, \gamma_{2}=\sqrt{{k_{x}{ }^{2}-\varepsilon_{d}{k_{0}}^{2}}^{2}}, k_{x}=\omega / v$, and $k_{0}=\omega / c$. At THz frequencies, graphene conductivity simplifies to $\sigma_{\mathrm{gp}}(\omega)=4 \alpha \epsilon_{0} c_{\overline{\hbar(\omega+i / \tau)}}$ on the condition that $E_{F} \gg k_{B} T$, where $\tau$ is the relaxation time, $T$ is the temperature, and $k_{B}$ is the Boltzmann constant [5]. Note that poles of the reflection coefficient $(\eta+\xi=-1)$ correspond exactly to the phase matching condition of Eq. (1), i.e., the excitation of GPs [21. As a result, $E_{x}$ exhibits resonances due to the excitation of GPs, as shown in Fig. 1(c), indicating that the EM fields near the graphene layer are considerably enhanced around GP resonant frequencies. From Eq. (2), the enhancement factor 

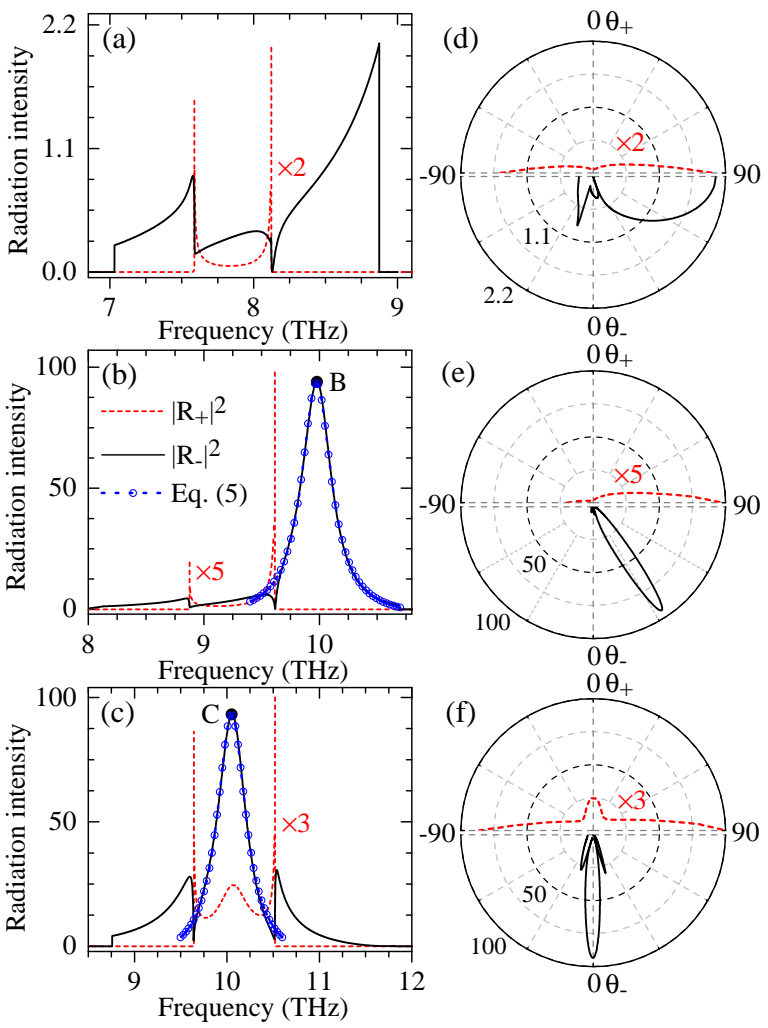

FIG. 3. (Color online) Radiation intensity $\left|R_{+}\right|^{2}$ toward the air side (red dashed lines) and $\left|R_{-}\right|^{2}$ toward the substrate side (black solid lines) versus frequency (left panels) and observation angle $\theta$ (right panels) for the setup shown in Fig. 2(a). Blue dotted lines with open circles represent the results of fitting to Eq. (5). The velocity of electron bunch is $v=0.034 c$ in (a) and (d), $0.04 \mathrm{c}$ in (b) and (e), and $0.0436 \mathrm{c}$ in (c) and (f). The modes B and C in (b) and (c) are related to those in Fig. 2, respectively.

is exactly $|t \eta|$, being about $\frac{2}{1+\varepsilon_{d}} \tau \omega_{\mathrm{gp}}$ at GP resonant frequencies. For example, at frequencies of 3 and $30 \mathrm{THz}$, $\tau \omega_{\text {gp }}$ can be about 11 and 110 with $\tau \sim 0.6$ ps respectively [22].

As shown, an electron bunch moving atop a graphene layer can efficiently excite GPs. However, the excited GPs cannot couple into free space due to the wavevector mismatch between GPs and free-space radiations. To transform GPs into free-space radiations, we consider a graphene layer situated on a periodic dielectric grating, as schematically shown in Fig. 2(a). The grating consists of $1 \mathrm{D}$ periodic grooves on a dielectric substrate. The groove has period $a$, thickness $h$, and filling fraction $f$. In the following discussions, the dielectric substrate has $\varepsilon_{\mathrm{g}}=11.6$, and other parameters are $a=1.3 \mu \mathrm{m}, h=$ $0.4 \mu \mathrm{m}, f=0.7$, and $b=0.1 \mu \mathrm{m}$.

The underlying physics for the transformation of excited GPs into free-space radiations stems from the fact that the wavevector mismatch can be compensated by

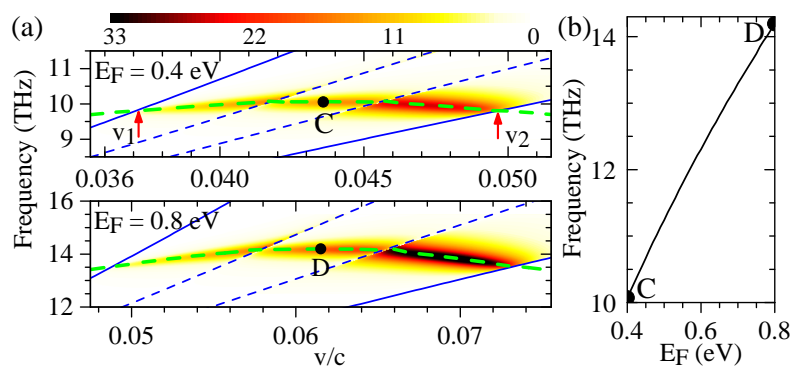

FIG. 4. (Color online) (a) Spectral density of radiated energy $d W_{-} / d \omega$ toward the substrate versus $v$ and frequency for a setup with $b=0$ as shown in Fig. 2(a). $d W_{-} / d \omega$ is of unit of $\rho^{2} / \epsilon_{0} c$ and plotted in color scale form. Green dashed lines are obtained from Eq. (1). Blue dashed (blue solid) lines are obtained from intersection points of red lines with blue dashed (blue solid) lines in Fig. 2(b). (b) Frequency of the excited GP at $k_{x}=0$ [see Fig. 2(b)] versus $E_{F}$. The corresponding $v$ can be calculated from $\omega /\left(k_{x}+2 \pi / a\right)=v$.

reciprocal lattice vectors of the grating, namely

$$
k_{\mathrm{gp}}+2 \pi m / a=\left\{\begin{array}{ll}
k_{0} \sin \theta_{+}, & z>0 \\
\sqrt{\varepsilon_{\mathrm{g}}} k_{0} \sin \theta_{-}, & z<0
\end{array},\right.
$$

where $m$ is the diffraction order of the grating and $\theta$ is the radiation angle with respect to $z$. Different branches of diffraction order $m$ represent band foldings. Consequently, the GP dispersion is now characterized by a well-defined band structure, as shown in Fig. 2(b), which is obtained numerically by a scattering matrix method [23. Bandgaps appear at the Brillouin zone center and boundaries due to multiple Bragg scatterings arising from the introduced periodicity. As a result, GP modes can reside above the light line, and therefore radiate into free space when excited by the moving electron bunch [Fig. 2(c)], giving rise to the so-called diffraction radiation [18, 24, 25. Note that there are two kinds of light lines, one for the air side and the other for the substrate side. The radiation patterns and directionality of excited GPs are determined by their positions in the Brillouin zone. In this study, we focus on GP modes in the first folded band $(m= \pm 1)$; GP modes in other bands can be analyzed similarly based on Eq. (3).

To characterize quantitatively the radiation induced by the moving electron bunch, we obtain the spectral density of radiated energy as [16]

$$
\frac{d}{d \omega} W_{ \pm}=\frac{\rho^{2}}{4 \pi \varepsilon_{0} c} \frac{\cos \left(\theta_{ \pm}\right)}{\sqrt{\varepsilon_{ \pm}}}\left|R_{ \pm}(\omega)\right|^{2} e^{-2 \gamma_{0} b}
$$

where the plus (minus) sign stands for the air (substrate) side, and $R$ is referred to as the radiation factor with $R_{+}\left(R_{-}\right)$being the reflection (transmission) coefficient of the structure [19].

Figures 3(a)-3(c) show the radiation intensity $\left|R_{ \pm}(\omega)\right|^{2}$ as a function of frequency. From Eq. (3), the radiation 
intensity is also plotted as a function of radiation angle $\left|R_{ \pm}(\theta)\right|^{2}$, shown in Figs. 3(d)-3(f). For $v=0.034 c$ [Fig. 3(a)], since the excited GP mode [mode $\mathrm{A}$ in Fig. 2(b)] lies below the light line of both air and substrate, it cannot contribute to free-space radiations. Only a continuum of Smith-Purcell (SP) radiation from the grating is observed toward both sides [13, 19. Also, the radiation is distributed over a broad angular range [Fig. 3(d)]. In contrast, for $v=0.0436 c$, prominent resonant peaks in the radiation spectra can be observed toward both the air and substrate sides [Fig. 3(c)], corresponding exactly to the excited GP mode [mode $\mathrm{C}$ in Fig. 2(b)]. Interestingly, the radiation is highly directional and normal to the grating [Fig. 3(f)]. Note that there exists still SP radiation toward both sides but with much lower intensity compared with that from the excited GP. When $v=0.04 c$ [Fig. 3(b)], a resonant peak positioned at the excited GP frequency [mode B in Fig. 2(b)] is observed only toward the substrate with its intensity dominating over that of SP radiation. From Fig. 3(e), highly directional radiation at an oblique angle is observed toward the substrate, whereas a broad angular emission of SP radiation occurs toward air.

To gain a deeper insight into the physics of the strongly enhanced radiation from excited GPs, we develop a selfconsistent electromagnetic theory of the coupling between moving electrons and GPs by assuming that the induced EM fields can be expressed in terms of GP modes around resonant frequencies $\omega_{\mathrm{gp}}$ 16. The theory can provide a closed-form expression for the radiation intensity as

$$
\left|R_{ \pm}(\omega)\right|^{2}=F \frac{\omega_{\mathrm{gp}}^{2}}{4 Q^{2}\left(\omega-\omega_{\mathrm{gp}}\right)^{2}+\omega_{\mathrm{gp}}^{2}} \frac{Q}{Q_{r, \pm}} \frac{\sqrt{\varepsilon_{ \pm}}}{\cos \left(\theta_{ \pm}\right)}
$$

where $F=\frac{2}{\pi} \frac{Q}{V / \lambda}, V$ is the generalized mode volume [26], $Q$ is the total quality factor, $Q_{r, \pm}$ is the radiative quality factor associated with the coupling of GPs into free space toward the air (substrate) side, and $\lambda=2 \pi c / \omega_{\mathrm{gp}}$. Note that $\left|R_{ \pm}(\omega)\right|^{2}$ exhibits a Lorentzian line shape, reaching a maximum of $F \frac{Q}{Q_{r, \pm}} \frac{\sqrt{\varepsilon_{ \pm}}}{\cos \left(\theta_{ \pm}\right)}$at resonant frequencies. It is found that both $Q$ and $\lambda / V$ can be of the order of 10 [27, and therefore the radiation enhancement arises from high $Q$-factors and small mode volumes of excited GPs.

To describe quantitatively the radiated energy, its spectral density as a function of $v$ and frequency at different $E_{F}$ is shown in Fig. 4(a). We only discuss the radiation toward the substrate since its intensity is much larger than that toward air. For a given $E_{F}$, the radiation from excited GPs occurs over a narrow frequency range, showing a weak dependence on $v$. This weak dependence stems from the weak dispersion of GP bands above the light line [21]. However, the radiation peak shows a strong dependence on $E_{F}$. From Fig. 4(b), the peak frequency varies roughly from 10 to $14.2 \mathrm{THz}$ as $E_{F}$ increases from 0.4 to $0.8 \mathrm{eV}$. Note that the spectral range

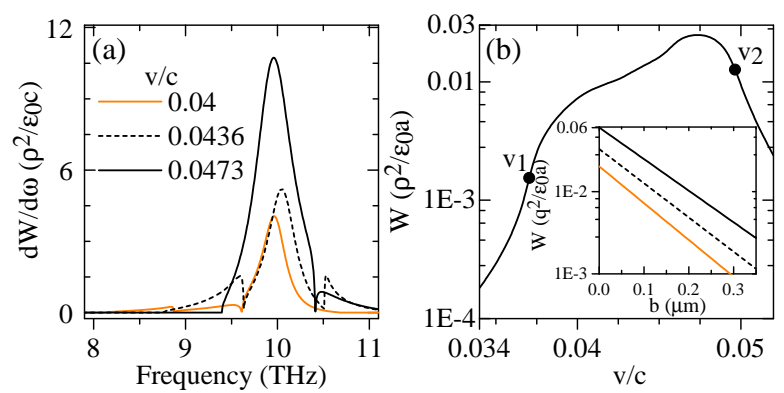

FIG. 5. (Color online) (a) Spectral density of radiated energy $d W_{-} / d \omega$ toward the substrate versus frequency, and (b) radiated energy $W_{-}$toward the substrate versus $v$ for a setup with $b=0.1 \mu \mathrm{m}$ as shown in Fig. 2(a). The inset to (b) plots the radiated energy $W_{-}$versus $b$ for different $v$ studied in (a). $v_{1}$ and $v_{2}$ in (b) correspond to those in Fig. 4(a), respectively.

of the radiation can be further tuned by engineering the grating structure [16].

By integrating the spectral density of Eq. (4) over frequency and radiation angle, the total radiated energy $W$ can be obtained, shown in Fig. 5(b) as a function of $v$. The radiated energy is dominate over the velocity range where the excited GPs contribute to the radiation. For example, for $v=0.0436 c$, the radiated energy is $W=1.26 \times 10^{-2} \rho^{2} / \epsilon_{0} a$, nearly two order of magnitude larger than $W=1.81 \times 10^{-4} \rho^{2} / \epsilon_{0} a$ for $v=0.034 c$, where only SP radiation occurs [Fig. 4(a)]. To evaluate quantitatively the radiated energy, we consider an electron bunch with $\rho=100 \mathrm{pC} / \mathrm{cm}$. The total radiated energy is estimated to be $W=1.099 \times 10^{-3} \mu \mathrm{J} / \mathrm{cm}^{2}$ for $v=0.0436 c$, corresponding to a peak power of $1.31 \mathrm{~kW} / \mathrm{cm}^{2}$ for a radiation pulse of $0.841 \mathrm{ps}[28$. The peak value of $d W / d \omega$ is found to be $0.0123 \mu \mathrm{J} / \mathrm{cm}^{2} / \mathrm{THz}$ at $10.05 \mathrm{THz}$.

In our calculations, the induced GP radiation is mainly influenced by the impurity- and phonon-limited relaxation time $\tau=0.6 \mathrm{ps}$ [16, which is estimated for $E_{F}=0.4 \mathrm{eV}$ from the measured DC mobility $\mu=$ $1.5 \times 10^{4} \mathrm{~cm}^{2} / \mathrm{Vs}$ at room temperature 29 . While impurity scattering is the dominant factor limiting $\tau$ in lowquality graphene [30], $\tau$ can be improved in high-quality graphene which has been reported to achieve high mobility values, an order of magnitude larger than what is assumed in this work [2, 31, 32. On the other hand, graphene optical phonons significantly degrade $\tau$ for frequencies above $\omega_{\mathrm{oph}}=48.4 \mathrm{THz}[5,33$. Howerver, the $\mathrm{THz}$ frequency regime of interest is below $\omega_{\mathrm{oph}}$. Therefore, high-performance GP radiation could be realized in the $\mathrm{THz}$ regime.

We now consider the experimental implementation of our proposal. The fabrication of high-quality graphene and its integration with subwavelength dielectric gratings have already been demonstrated experimentally [29, 34 . While sub-ps electron bunches required in the $\mathrm{THz}$ 
regime can be obtained in the keV energy range with the state-of-the-art development of ultrafast pulsed electron sources by employing femtosecond lasers [35, continuous electron beams from low-voltage electron microscopes could also be employed to realize the effect shown in this letter 24]. By taking advantage of high resolution of electron microscopes, electron beams can be directed parallel to the surface of a system over a distance on the nanometer scale [24, 36. Although charge density in sub-ps electron bunches with good beam quality may be limited, electron bunch trains with high repetition rate could be used to further increase the radiation intensity 37.

In conclusion, we have shown that GPs excited by uniformly moving electrons with low electron energies can give rise to $\mathrm{THz}$ radiations with strongly enhanced intensity due to low losses and high confinement of GPs. Importantly, the radiation frequency can be tuned by varying $E_{F}$ via electrostatic gating or chemical doping. In addition, the possibility of using low-energy electrons could overcome the size limit of conventional free-electron $\mathrm{THz}$ sources requiring high-energy electrons [12]. Therefore, our results could open up the possibility of developing miniature free-electron $\mathrm{THz}$ radiation sources with high tunability based on graphene plasmonics.

This work was supported by the 973 Program (Grant Nos. 2013CB632701 and 2011CB922004). The research of J.Z. is further supported by the NSFC.

* phystrzhan@gmail.com

$\dagger$ jzi@fudan.edu.cn

[1] F. Bonaccorso et al., Nat.Photon. 4, 611 (2010).

[2] A. K. Geim et al., Nat. Mater. 6, 183 (2007).

[3] B. Wunsch et al., New J. Phys. 8, 318 (2006); E. H. Hwang and S. Das Sarma, Phys. Rev. B 75, 205418 (2007).

[4] W. L. Barnes et al., Nature (London) 424, 824 (2003).

[5] M. Jablan et al., Phys. Rev. B 80, 245435 (2009); F. H. L. Koppens et al., Nano Lett. 11, 3370 (2011).

[6] A. N. Grigorenko et al., Nat. Photon. 6, 749 (2012).

[7] A. Vakil and N. Engheta, Science 332, 1291 (2011).

[8] L. Ju et al., Nat. Nanotechnol. 6, 630 (2011).

[9] S. Thongrattanasiri et al., Phys. Rev. Lett. 108, 047401 (2012).

[10] S. Thongrattanasiri et al., Phys. Rev. Lett. 110, 187401
(2013).

[11] M. Tonouchi, Nat. Photon. 1, 97 (2007).

[12] G. P. Gallerano, and J. A. Valdmanis, in Proceedings of the 2004 FEL Conference (Trieste, Italy, 2004), pp. 216.

[13] S. J. Smith et al., Phys. Rev. 92, 1069 (1953).

[14] A. M. Cook et al., Phys. Rev. Lett. 103, 095003 (2009).

[15] P. G. O'Shea et al., Science 292, 1853 (2001).

[16] See Supplemental Material at for further details on the derivation of Eqs. (2), (4), and (5), tunability of induced GP radiation by engineering the grating, a discussion of the influence of the relaxation time, and electrostatic effects on the moving electrons for electrostatic doping.

[17] L. D. Landau, E. M. Liftshitz, and L. P. Pitaevskii, Electrodynamics of Continuous Media (Pergamon, 1984).

[18] F. J. García de Abajo, Rev. Mod. Phys. 82, 209 (2010).

[19] P. M. van den Berg, J. Opt. Soc. Am. 63, 689 (1973).

[20] S. Yamaguti et al., Phys. Rev. B 66, 195202 (2002); F. J. García de Abajo et al., Phys. Rev. B 67, 125108 (2003).

[21] T. R. Zhan et al., J. Phys. Condens. Matter 25, 215301 (2013).

[22] At $\omega=\omega_{\mathrm{gp}},\left|E_{x}(z=0)\right| \simeq \frac{\rho}{c \varepsilon_{0}\left(1+\varepsilon_{d}\right)} \frac{\tau \omega_{\mathrm{gp}}}{k_{0} / \gamma_{0}} e^{-\gamma_{0} b}$. Note that $1 / \gamma_{0}$ is the GP decay length along $z$, and $k_{0} / \gamma_{0} \simeq v_{\mathrm{gp}} / c$ is of the order of $0.1 \sim 0.01$. Hence, the excitation of GPs by moving electrons also benefits from the strong confinement of GPs.

[23] T. R. Zhan et al., Phys. Rev. B 86, 165416 (2012).

[24] G. Adamo, et al., Phys. Rev. Lett. 103, 113901 (2009); G. Adamo et al., J. Opt. 12, 024012 (2010).

[25] M. I. Bakunov et al., Opt. Express 17, 9323 (2009); S. Liu et al., Phys. Rev. Lett. 109, 153902 (2012).

$[26] V \equiv \frac{\int\left[\varepsilon_{0} \varepsilon(\mathbf{r})|\tilde{\mathbf{E}}|^{2}+\mu_{0}|\tilde{\mathbf{H}}|^{2}\right] d x d z}{2 \varepsilon_{0}\left|\int \tilde{\mathbf{E}} x_{x}(z=0) e^{i k_{x} x} d x\right|^{2}}$ where $k_{x}=\omega / v$ and $\{\tilde{\mathbf{E}}, \tilde{\mathbf{H}}\}$ is the field distribution of GP modes 16. Note that both transverse-magnetic polariztaion and strong confinement of GPs are necessary for achieving a small $V$.

[27] By fitting the numerical results to Eq. (5) [16], it is obtained that, when $\tau=0.6 \mathrm{ps}, Q=25.4(20.5), \lambda / V=$ $6.9(13.1)$, and $Q / Q_{r,-}=0.21(0.31)$ for $v / c=0.0436$ (0.0473).

[28] Pulse duration of the radiation is estimated from the spectral bandwidth of radiation peaks.

[29] Y. Hao et al., Science 342, 720 (2013).

[30] J. H. Chen et al., Nat. Nanotechnol. 3, 206 (2008).

[31] K. I. Bolotin et al., Solid State Commun. 146, 351 (2008).

[32] C. R. Dean et al., Nat. Nanotechnol. 5, 722 (2010).

[33] H. Yan et al., Nat. Photon. 7, 394 (2013).

[34] W. Gao et al., Nano Lett. 13, 3698 (2013).

[35] A. J. McCulloch et al., Nat. Commun. 4, 1692 (2013); P. Hommelhoff et al., Phys. Rev. Lett. 96, 077401 (2006); B. J. Siwick et al., Science 302, 1382 (2003).

[36] W. E. King et al., J. Appl. Phys. 97 (2005).

[37] S. E. Korbly et al., Phys. Rev. Lett. 94, 054803 (2005). 